\title{
Elective Cesarean Delivery
}

National Cancer Institute

\section{Source}

National Cancer Institute. Elective Cesarean Delivery. NCI Thesaurus. Code C114141.

A cesarean delivery scheduled at a patient's request or provider preference without medical or obstetric indications. 